\title{
Prototyping of a Highly Performant and Integrated Piezoresistive Force Sensor for Microscale Applications
}

\author{
Bilal Komati, Joël Agnus, Cédric Clévy and Philippe Lutz \\ FEMTO-ST Institute, AS2M department, \\ Université de Franche-Comté/CNRS/ENSMM/UTBM, \\ 24 rue Savary, 25000 Besançon, France. \\ E-mail: bilal.komatiefemto-st.fr, Joel.Agnuseens2m.fr, \\ cclevy@femto-st.fr, philippe.lutz@femto-st.fr
}

\begin{abstract}
In this paper, the prototyping of a new piezoresistive microforce sensor is presented. An original design taking advantage on both mechanical and bulk piezoresistive properties of silicon is presented and enables to easily fabricate a very small, large range, high sensitivity with high integration potential sensor. The sensor is made of two silicon strain gages for which widespread and known microfabrication processes are used. The strain gages present a high gage factor which allow a good sensitivity of this force sensor. The dimensions of this sensor are $700 \mu \mathrm{m}$ in length, $100 \mu \mathrm{m}$ in width and $12 \mu \mathrm{m}$ in thickness. These dimensions make its use convenient with many microscale applications notably its integration in a microgripper. The fabricated sensor is calibrated using an industrial force sensor. The design, microfabrication process, and performances of the fabricated piezoresistive force sensor are innovative thanks to its resolution of $100 \mathrm{nN}$ and its measurement range of $2 \mathrm{mN}$. This force sensor presents also a high signal to noise ratio, typically $50 \mathrm{~dB}$ when a $2 \mathrm{mN}$ force is applied at the tip of the force sensor.
\end{abstract}

\section{Introduction}

At the microscale, sensors are necessary in order to measure what is happening, to understand microsystems behaviors and to control them. Notably, the force measurement is very important because contact forces and dynamics are predominant at the microscale. It could be used in many applications such as biology [1, 2], biomedical [3], microgrippers [4], microassembly [5], micromanipulation [6], etc. Integrating force sensors in microsystems improves and facilitates the tasks by providing a force feedback which enables force control of the system [7, 8]. The force measurement at the microscale needs not only to integrate the sensors on or inside the system itself but also to perform measurement the closest to the area of interest [9]. This avoids measuring what is happening outside the system itself, reduces the parameter variations of the system and reduces the noise which are predominant factors at the microscale [10].

Furthermore, the sensor should be able to measure forces present and predominant at the microscale such as surface forces, van der Waals forces, capillary forces, contact forces, manipulation forces, etc. These forces are in the range of hundreds of $\mathrm{nN}$ up to several $\mathrm{mN}$ 
$[11,12,7,13]$. However, few solutions exist to fabricate a force sensor in this measuring range and most of the works present force sensors which are difficult to be integrated in a small free size. The lack of works is essentially due to the design constraints, sensor's performances required (size, resolution, dynamics, microfabrication aspects, etc) and the need of multidisciplinary skills (design, simulation, microfabrication, electronics, characterization, etc).

Thus, the aim of this work is to propose a force sensor principle which could be used in many domains and could be integrated within most of microsystems and to develop technology processes to fabricate this force sensor.

Several principles for force sensing exist: capacitive, resonant structure, piezoelectric and piezoresistive. Among these working principles, capacitive sensors $[14,15,16]$ use the capacitance change of a compliant silicon structure. This kind of sensor is quite sensitive but often needs up to several centimeters square of sensing surface. The main limitations of the capacitive force sensors are their relative big size, their complicated microfabrication process due to the need of insulation between electrodes and the difficulty of their integration. Another principle uses the frequency shift of a resonant structure [17, 18]. Even if this principle works quite well, it is usually difficult to integrate it into a microsystem and the oscillation may disturb the task to perform (gripping task for example).

Direct piezoelectric effect is also used as force sensor [19] by the mean of charge amplifiers. This latter integrates the electrical charges which limits the measurement of dynamic forces above several Hertz.

The most used principle for microscale applications is the piezoresistive effect that exploits the resistance change according to the deformation of a compliant structure. The piezoresistive sensors are widely used in many applications due to their small scale, easy integration, convenient readout method and high measurement dynamics. They are widely used as sensing elements in pressure sensors [20], accelerometers [21], flow sensors [22], Atomic Force Microscopy (AFM) [23].

There are several approaches for fabricating piezoresistive force sensors with high performances. Some piezoresistive force sensor using metal materials are presented in $[24,25]$ and others using silicon are presented in [26, 27, 28]. The use of silicon to fabricate force sensors is common because it benefits from the skills earned of using silicon for the microfabrication of MEMS devices. Indeed, the silicon is a widespread material used for the fabrication of many types of MEMS due to the standard microfabrication processes that can be used, its relatively low production cost and its mechanical and structural properties. In addition, silicon presents another advantage once it is used for the fabrication of force sensors because its gage factor is among the highest compared to other materials which provides better sensitivity and performances of the sensor.

In most of existing works, the sensor is used to measure forces perpendicular to the microfabrication plane. However, many MEMS structures exhibit in-plane motions that need to measure the resulting in-plane forces. In this case, surface or volume strain gages could be used to sense the strain dissymmetry in the plane of the MEMS structure. Thus, this work proposes the use of bulk strain gages to simplify the fabrication process of the sensor and 
gives the possibility to integrate it in most of microsystems without additional and complex processes like epitaxial deposition of thin Silicon layers or ion implantation to obtain doped gauges.

A work using bulk piezoresistive force sensor has been presented in [28] where the authors designed a microgripper with an integrated piezoresistive force sensor. The focus of the paper is the design of a thermal actuator where some preliminary results of the force sensor are presented. The objective of our paper is to optimize the design of such a force sensor based on the bulk strain gauges, taking advantage of both mechanical and structural properties as well as piezoresistive properties of silicon. As a result, a new prototype of fabrication, using a low cost, simple, known, repeatable and high throughput microfabrication process is proposed and the sensor's performances are tested.

The paper is organized as follows. The piezoresistive force sensor, specifications, measurement principle and its design are presented in section 2. The simulations parameters used are detailed in section 3. The complete microfabrication process is presented in section 4. The calibration of the piezoresistive force sensor is performed and presented in section 5 including the experimental setup used for the calibration and the performance of the sensor are studied in this section. Section 6 concludes the article.

\section{Piezoresistive Force Sensor Theory and Design}

In this section, the specifications of the force sensor are defined, the piezoresistive force sensing measurement theory is revised and the force sensor design is proposed.

\subsection{Force Sensor Specifications}

In this section, the specifications of the force sensor are presented. The main parameters to take into consideration for the design are:

(i) the small free size to integrate sensors:

In many complex microsystems a lot of components should be integrated in a small operation area to perform a precise task such as microrobotic systems [7], actuators, micromirrors [29], cameras, platforms [5], etc. Thus, the force sensor should be as small as possible in order to be able to integrate it in such type of microsystems and in order to perform the measurement as close as possible of the area of interest. The dimensions of the force sensor is then fixed to be less than $1000 \times 100 \times 20 \mu \mathrm{m}^{3}$.

(ii) the resolution and the maximal force sensing range:

As already discussed, the sensing range of the force sensor is chosen from several hundreds of $\mathrm{nN}$ to several $\mathrm{mN}$.

\subsection{Piezoresistive Force Sensor Principle}

In this section, the piezoresistive force sensor principle of measurement is presented in two steps. First, a bulk sensor is presented and second some cavities are added to the bulk 
piezoresistive force sensor which increases the sensitivity of the force sensor and avoid electrical contact between strain gages.

2.2.1. Cantilever with bulk silicon strain gages The structure of the cantilever with bulk silicon strain gages is given in Figure 1-a. The strain gage is across all the volume of the cantilever. The strain $\varepsilon$ inside the strain gages (shown in Figure 1-a) is defined in function of the relative variation of the length by the following [30]:

$$
\varepsilon=\frac{d L}{L}
$$

The piezoresistive change in the strain gage resistance $\frac{d R}{R}$ can be calculated from the gage factor $G$ and change in strain $\varepsilon$ by:

$$
\frac{d R}{R}=G \cdot \varepsilon
$$

where $R$ is the resistance of the bulk strain gage.

The sensitivity of the strain gage increases by increasing its gage factor $(G)$. There are metal gages mainly sensitive to changes in geometry $(G=2-5)$ [31] and silicon strain gages that has a large piezoresistive effect $(G>100)$ [32]. Thus, integrating silicon strain gages into microdevices is commonly used for the fabrication of microforce sensors.
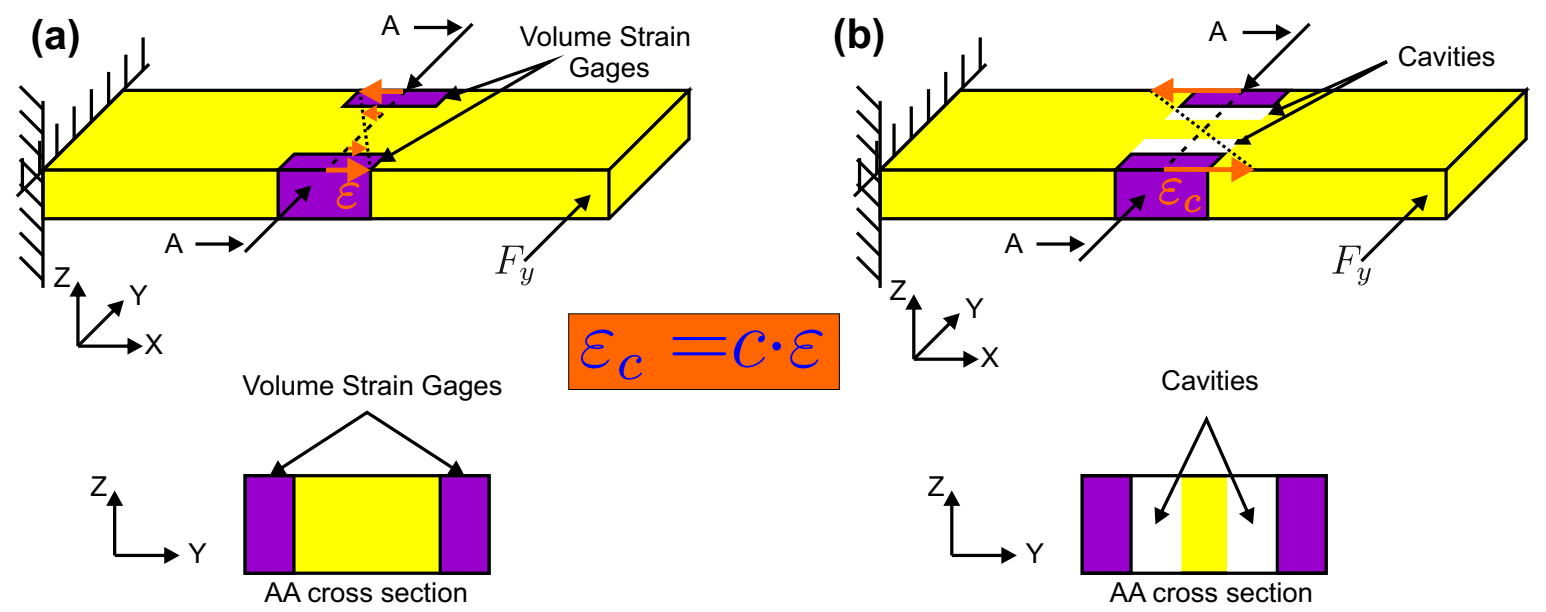

Figure 1. Strain distribution inside a cantilever with two bulk strain gages where a force is applied at its tip: (a) no cavity and (b) with cavities.

2.2.2. Cantilever with bulk strain gages and with two cavities The structure of the cantilever with bulk silicon strain gages with cavities is given in Figure 1-b. According to [26], adding a cavity before the strain gage increases the sensitivity of the sensor due to the amplification of the strain in the strain gage. Hence, in the final design of the force sensor is composed of 
three parallel beams as shown in Figure 1. Using (1), the strain, in this case could be written as:

$$
\varepsilon_{c}=\frac{d L}{L}=c \cdot \varepsilon
$$

where $\varepsilon_{c}$ is the strain inside the strain gages after adding cavities (shown in Figure 1-b) and $c$ is a constant which adds the effect of adding the cavities and $\varepsilon$ is the strain given in (1). Using [26], $c$ is bigger than $1(c>1)$ and its value will be determined in section 3 . Using (2) and (3), the piezoresistive change in the strain gage resistance after adding cavities is given by:

$$
\frac{d R}{R}=c \cdot G \cdot \frac{d L}{L}=c \cdot G \cdot \varepsilon
$$

Hence, the complete sensitivity of the force sensor is increased by a factor of $c$ relatively to the force sensors with bulk or surface strain gages.

\subsection{Piezoresistive Force Sensor Design}

In this section the design of the force sensor is presented. The use of silicon is chosen because it is a widespread material used in MEMS structures and it presents a good gage factor $G$. The design proposed benefits from the mechanical and structural properties of the silicon as well as its bulk piezoresistive properties. Thus, as shown in Figure 2-(a), a structure, composed of three parallel beams where the two side beams are sensitive to stresses exerted by the application of a force $F_{y}$, is proposed.

Without applying any force to the tip of the force sensor, the two gage resistances are identical and equal to $R_{o}$. The application of a positive force $F_{y}$ causes a contraction of the left beam with a decreased resistance $-d R$, and an extension of the right beam with an increased resistance $d R$. After applying positive force $F_{y}$, the left and right gages resistances become respectively $R_{1}=R_{o}-d R$ and $R_{2}=R_{o}+d R$. The central beam allows the passage of the common electrode (point A in Figure 2 of the Wheatstone bridge) and allows for better symmetry of deformation of the sensitive beams.

Antagonistic resistances $R_{1}$ and $R_{2}$, which are sensitive to $F_{y}$, are mounted in a Wheatstone

(a)

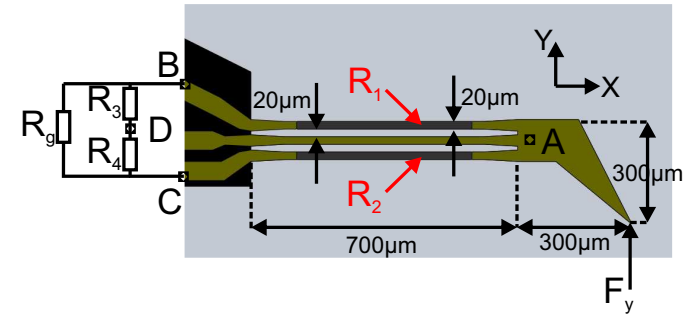

(b)

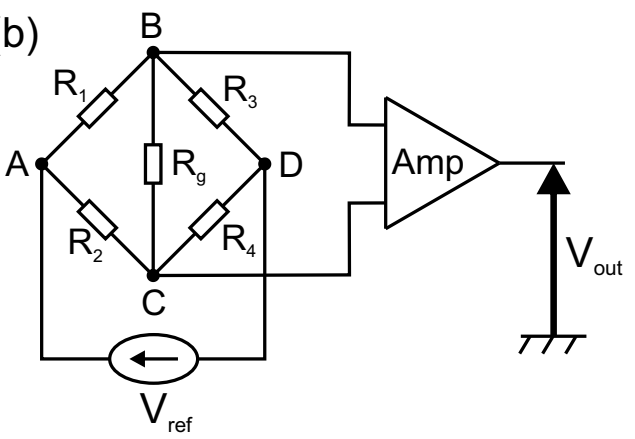

Figure 2. (a) Sensing beam structure and (b) its Wheaststone bridge

bridge. The latter is connected to a differential amplifier, Amp, to obtain a voltage image, $V_{\text {out }}$, 


\begin{tabular}{|c|c|}
\hline Parameter & Value \\
\hline Young Modulus & $169 \mathrm{GPa}$ \\
\hline Poisson ratio & 0.278 \\
\hline Electrical Resistivity & $1000 \Omega \cdot \mathrm{m}$ \\
\hline$\pi_{11}$ & $6.6 \times 10^{-11} \mathrm{~Pa}^{-1}$ \\
\hline$\pi_{12}$ & $-1.1 \times 10^{-11} \mathrm{~Pa}^{-1}$ \\
\hline$\pi_{44}$ & $138.1 \times 10^{-11} \mathrm{~Pa}^{-1}$ \\
\hline
\end{tabular}

Table 1. Silicon parameters used in the simulations using COMSOL MULTIPHYSICS.

of the applied force $F_{y}$ as shown in Figure 2-(b). $V_{r e f}$ is the voltage applied to the Wheatstone bridge.

\section{Simulations}

In this section, simulations are performed to design the force sensor and to test its predicted performances. The simulations are performed using COMSOL MULTIPHYSICS. The parameters used in the simulations for the design of the piezoresistive force sensor are detailed in Table 1. After considering the specifications of the dimensions and the force sensing range of the force sensor presented in section 2.1 and using the design presented in Figure 2-(a), many simulations have been done to determine the final dimensions and parameters of the force sensor. The thickness of the force sensor is fixed to $12 \mu \mathrm{m}$ by choosing the thickness of the device layer of the wafer to be used for the microfabrication. The width is fixed to $100 \mu \mathrm{m}$. Then, the length of the sensor is changed to meet the presented specifications. Thus, the dimensions of the force sensor are fixed to be $700 \mu \mathrm{m}$ in length, $100 \mu \mathrm{m}$ in width and $12 \mu \mathrm{m}$ in thickness. Each gage of the sensor has $20 \mu \mathrm{m}$ of width. The sensor's expected resolution is $500 \mathrm{nN}$, its complete sensing range is $2 \mathrm{mN}$ and its stiffness is $125 \mathrm{~N} / \mathrm{m}$. The resistance for each of the silicon strain gage without any applied stress, $R_{o}$, is $3 \mathrm{k} \Omega$. The coefficient $c$ presented in (4) is tested in simulations and is equal to $1.25(c=1.25)$ which means that the sensitivity of the force sensor is increased $25 \%$ by adding the cavities to the design. The simulation results will be compared with the experimental results in section 5 .

The complete design of the force sensor and its dimensions are given in Figure 2-(a).

In Figure 3, the strain distribution along the two strain gages of the adopted system is shown. The strain distribution variation is small and the strain distribution could be considered as constant along all the strain gage.

\section{Microfabrication}

In this section, a microfabrication process to fabricate the presented force sensor is proposed. As already said, the silicon is a widespread material used for the fabrication of many types of MEMS due to the standard microfabrication processes that can be used, its relatively low cost production and its mechanical and structural properties. In addition, considering design 


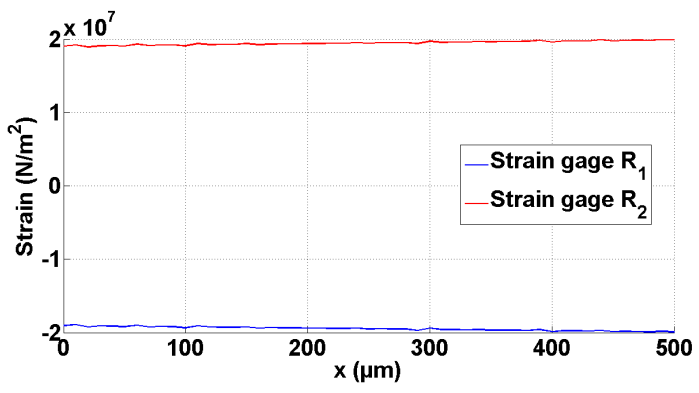

Figure 3. Strain distribution along $x$ axis for the two strain gages.

parameters and material requirements, silicon has been chosen for new force sensors due to its piezoresistive proprieties, its high strain gage factor and its high Young's Modulus. SOI wafers, combined with appropriate microfabrication processes, enable the fabrication of this kind of piezoresistive microforce sensor with adequate quality to obtain good sensor performances. Indeed, each silicon layer of the wafers can be separately etched: the handle layer $(350 \mu \mathrm{m})$ for the base of the finger and the device layer $(10 \mu \mathrm{m})$ for the gripping beam. Some dry and wet etching processes were considered, and finally DRIE (Deep Reactive Ion Etching), with BOSCH process was chosen for many reasons. The DRIE process is often used for many MEMS microfabrication process due its capability of deeply etching the silicon with a good anisotropy and to the speed of etching (around $6 \mu \mathrm{m} / \mathrm{min}$ ) that the BOSCH process provides. In addition, the etching side has a particular roughness, called scalloping, as a result of the way of guaranteeing etching anisotropy. This roughness presents an interest in many applications like gripping because it reduces the gripping surface between silicon force sensor and manipulated micro-objects [33].

The microfabrication flowchart presented in Figure 4 was used to manufacture the microforce sensor. Ti can be resumed by the following steps:

1) SOI wafer is used to start the fabrication. The wafer is composed of a $10 \mu \mathrm{m}$ device layer of silicon, $1 \mu \mathrm{m}$ of silicon oxide layer and $350 \mu \mathrm{m}$ handle layer of silicon (figure 4.1),

2) the wafer is thermally oxidized to create $1 \mu \mathrm{m}$ thick $\mathrm{SiO}_{2}$. The $\mathrm{SiO}_{2}$ is, then, etched in a BHF solution (Buffered Hydrofluoric Acid also called BOE for Buffered Oxide Etch) using photolithographically patterned photoresist (figure 4.2),

3) this step is a critical step in the microfabrication process, it is used to establish electric contact between the silicon piezoresistive gage and the external electrodes. It is formed by sputtering an aluminium layer on the silicon device layer and annealing it at a specific temperature in order to diffuse the aluminium atoms into the silicon. The diffusion of the aluminium atoms into the silicon determines the contact resistance which should be the smallest possible to enable good performance of the the sensor. Usually, high temperatures and long annealing time are used to diffuse the alumium atoms. However, this will decrease the sensitivity of the force sensor by decreasing the strain gage resistance. Indeed, the choice of the parameters in the annealing process is a critical step of the microfabrication process used. Finally, the ohmic contact is performed by 
sputtering $200 \mathrm{~nm}$ of aluminium, annealing it at $600^{\circ} \mathrm{C}$ for $1 \mathrm{~h}$ and etching the aluminium using photolithographically patterned photoresist (figure 4.3),

4) $800 \mathrm{~nm}$ of aluminium are patterned by lift-off to realize the electric connections on the device (figure 4.4),

5) Fifthly, the handle layer is etched using DRIE after sputtering $200 \mathrm{~nm}$ of aluminium to realize a mask for the DRIE on the handle layer (figure 4.5),

6) the device layer is etched using DRIE process using masks of $\mathrm{SiO}_{2}$ and aluminium (figure 4.6),

7) the process is finished by a top side RIE etching of the $\mathrm{SiO}_{2}$ (figure 4.7).

More than $95 \%$ of structures were fully operational. The $5 \%$ missing force sensors are due to a problem of temperature homogenity in the oven. Figures 5 and 6 show some SEM pictures at the end of the microfabrication process. Figure 5 shows the complete force sensor view while figure 6-(a) and (b) shows respectively the side and the top view of the clamping part of the force sensor to the rigid part (handle layer). The two figures show also the ohmic contact part $\left(3^{r d}\right.$ step in the microfabrication flowchart presented in figure 4). Figure 6-(a) shows also the scalloping due to the back side DRIE etching $\left(5^{\text {th }}\right.$ step in the microfabrication flow chart). Figure 6-(c) and (d) show respectively the side and the top view of a sensitive silicon beam. In figure 6-(c), the scalloping effect due to the front side DRIE is present $\left(6^{\text {th }}\right.$ step of the microfabrication flowchart).

The dimensions obtained after the fabrication are measured using SEM pictures as in figure 6. The sensor's length in the design is $700 \mu \mathrm{m}$ while the measurements using SEM showed that the sensor's length is $702.8 \mu \mathrm{m}$ with a fabrication error of $0.4 \%$. The width of the sensor's gages in the design is $20 \mu \mathrm{m}$ while the measurements using SEM showed that it is $20.05 \mu \mathrm{m}$ with a fabrication error of $0.25 \%$. The thickness of the device layer of the SOI wafer used in fabrication is $10 \pm 1 \mu \mathrm{m}$. As shown in figure $4,1 \mu \mathrm{m}$ of $\mathrm{SiO}_{2}$ is fixed on the device layer. In addition, $1 \mu \mathrm{m}$ of $\mathrm{SiO}_{2}$ is present on the internal layer of the SOI wafer. Adding the three layers, the theoretical thickness of the force sensor is $12 \pm 1 \mu \mathrm{m}$. After measurements, the thickness is measured to be $12.24 \mu \mathrm{m}$ which means $2 \%$ of error in the thickness to the model due to the uncertainity of the device layer thickness by construction.

\section{Sensor Calibration}

In this section, the sensor characteristics are investigated. First, an experimental setup used for the sensor calibration is proposed. Then, several tests are performed to determine the piezoresistive force sensor's stiffness, its sensitivity, its signal to noise ratio and its resolution.

\subsection{Experimental Setup}

An experimental setup is proposed to calibrate the piezoresistive microforce sensor (PiezoFS) (see Figure 7). The objective of this section is to determine the force sensor characteristics especially the stiffness, the sensitivity, the SNR (Signal to noise ratio) and the resolution of the 
1. SOI Wafer (Si $\left.350 \mu m-\mathrm{SiO}_{2} 1 \mu \mathrm{m}-\mathrm{Si} 10 \mu \mathrm{m}\right)$

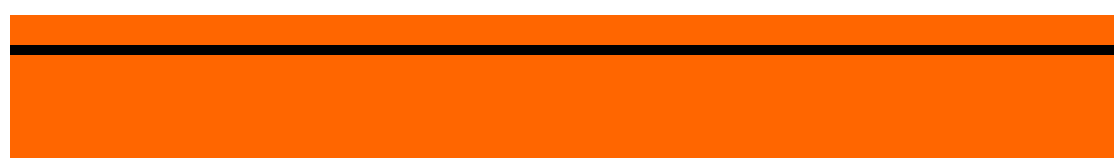

2. Thermal oxydation $\left(\mathrm{SiO}_{2} 1 \mu \mathrm{m}\right)$, photolithography and front side BHF opening (for ohmic contact and D-RIE mask)

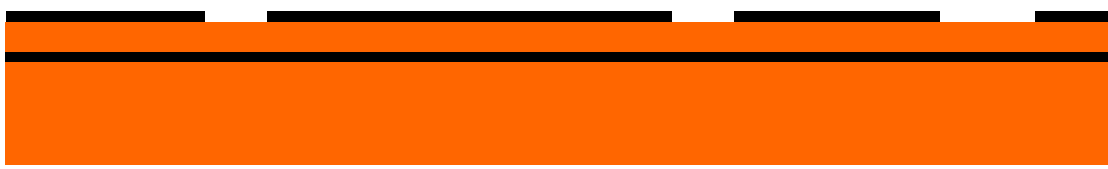

3. Ohmic contact patterning: front side Al sputtering $(200 \mathrm{~nm})$, annealing $\left(600^{\circ} \mathrm{C} / 1 \mathrm{~h}\right)$, photolithography and $\mathrm{Al}$ etching

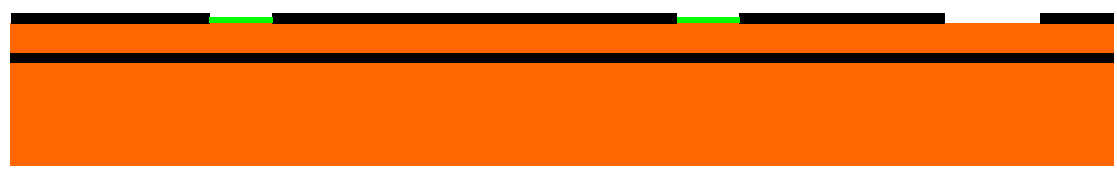

4. Electrodes patterning: front side Al lift-off (800nm)

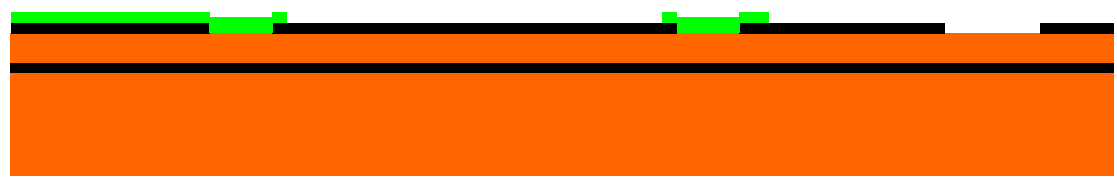

5. Back side Al sputtering (200nm), photolithography and D-RIE etching

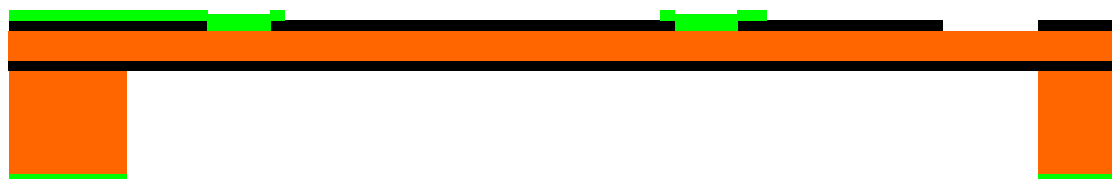

6. Front side D-RIE etching $\left(\mathrm{SiO}_{2}\right.$ and $\mathrm{Al}$ masks $)$

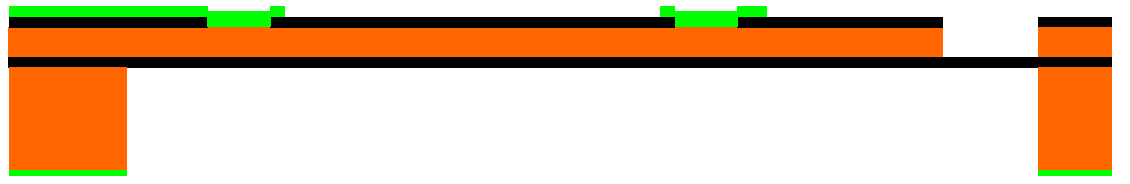

7. Front side RIE etching of $\mathrm{SiO}_{2}$ internal layer
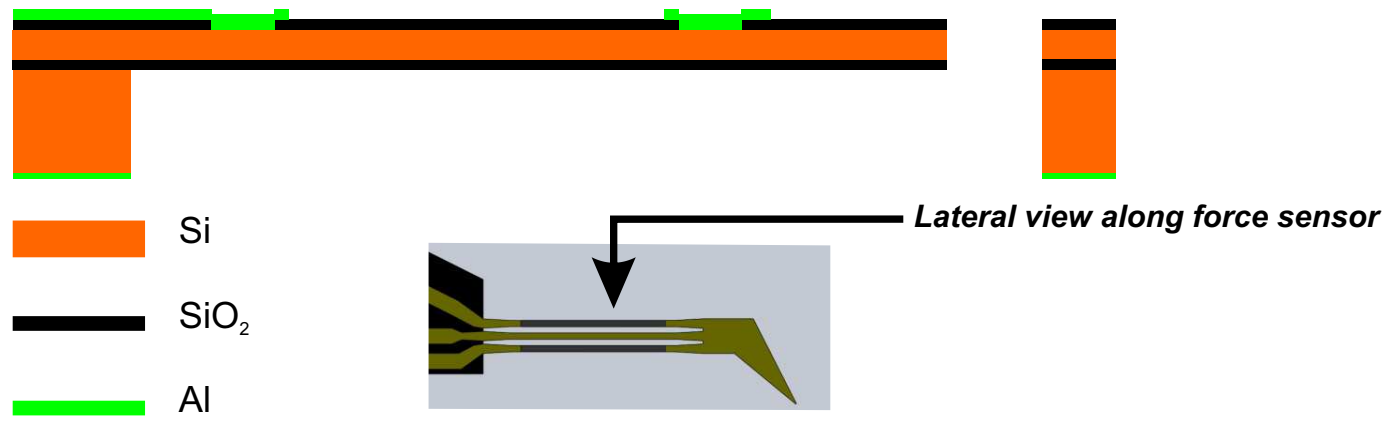

Figure 4. Microfabrication process flowchart.

PiezoFS. Using a Wheatstone bridge, the resistance variation of the gages of the PiezoFS are converted into voltage in order to perform the acquisition signal of the PiezoFS. An industrial and calibrated force sensor (CalibFS) is used to calibrate the PiezoFS. The CalibFS is a force sensor FT-S270 from FemtoTools with a measuring range of $2000 \mu \mathrm{N}$ and a resolution of $0.4 \mu \mathrm{N}$. The CalibFS comprises a probe tip, of $3 \mathrm{~mm}$ in length and $50 \mu \mathrm{m}$ in thickness, that moves along its main direction once a force is applied at its tip. The displacement is 


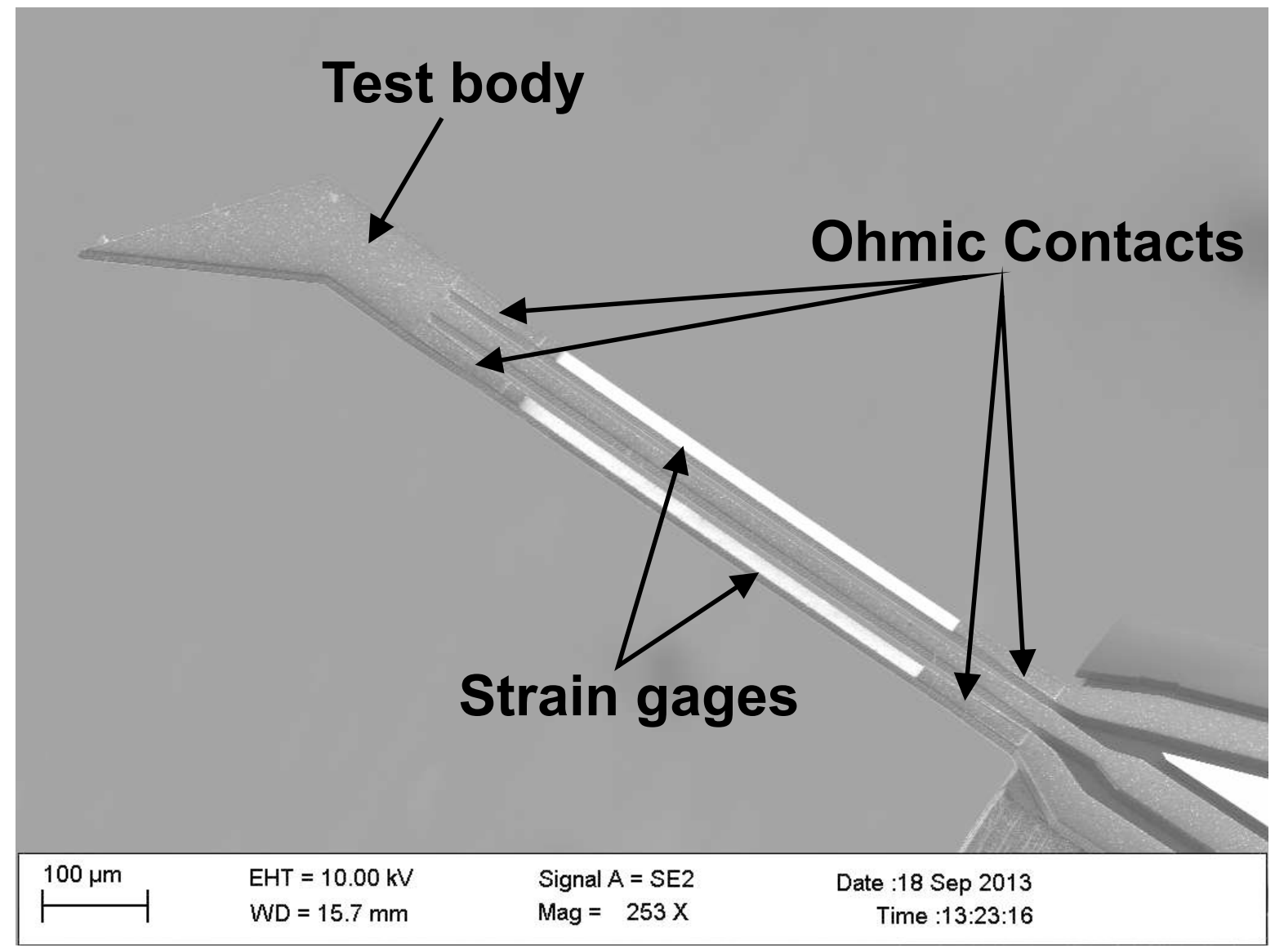

Figure 5. SEM picture of the complete force sensor showing the strain gages the ohmic contact and the test body.

converted into a voltage thanks to a capacitive variation measured by a dedicated circuit. The complete CalibFS with electric connections and mechanical support has dimensions of around $4 \mathrm{~cm} \times 2.5 \mathrm{~cm} \times 1.5 \mathrm{~cm}$. The CalibFS is mounted on a microrobotic structure composed of a fine positioning stage and rotation stage. The fine positioning stage is a P-611.3 NanoCube with $100 \mu \mathrm{m}$ range and $1 \mathrm{~nm}$ resolution. The rotation stage is a SmarAct SR-3610-S with 1.1 $\mu^{\circ}$ resolution is used to adjust the perpendicularity of contact between the PiezoFS and the CalibFS. These two motion devices are equipped with sensors and are closed loop controlled. The positioning stage and the voltage acquisition of the two force sensors are performed via a dSpace 1104 acquisition board with a sampling frequency of $10 \mathrm{kHz}$.

The calibration of the PiezoFS is done by moving the CalibFS, which is fixed to the fine positioning stage, into contact with the PiezoFS and then moved it in the opposite direction to separate contact along the Y direction of the figure 2. The output voltage of the PiezoFS, the force measurement of the CalibFS and the position measurement of the internal sensor of the fine positioning stage are saved and considered in order to determine the PiezoFS characteristics. 


\section{Ohmic Contacts}

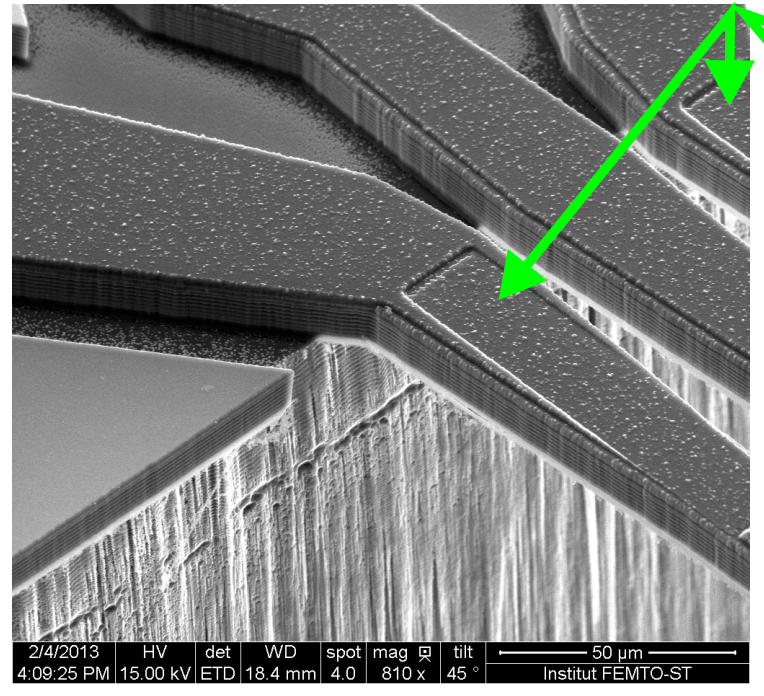

(a)

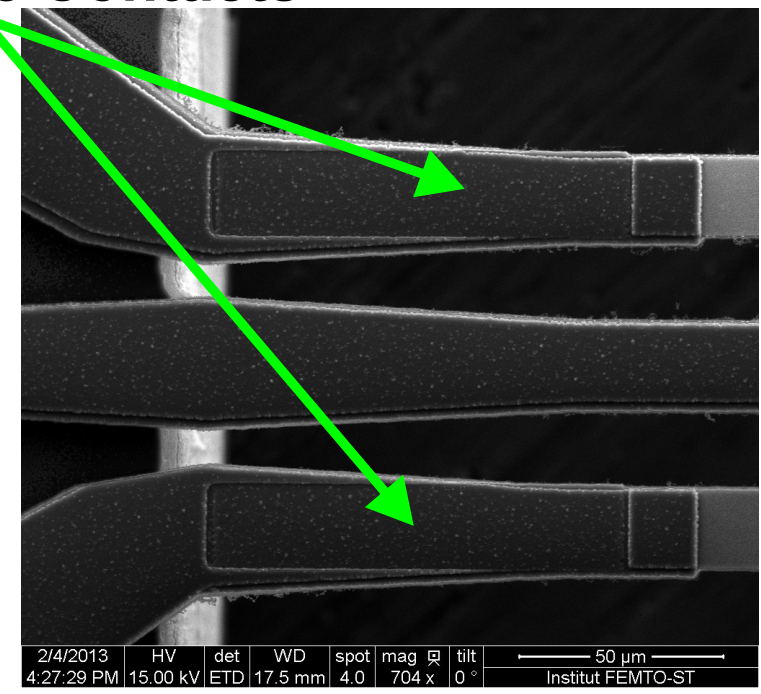

(b)

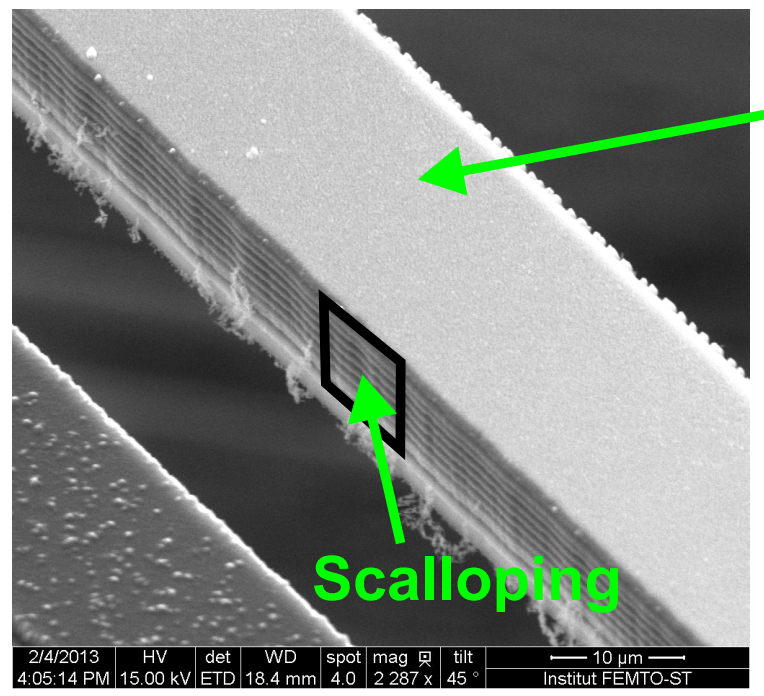

(c)

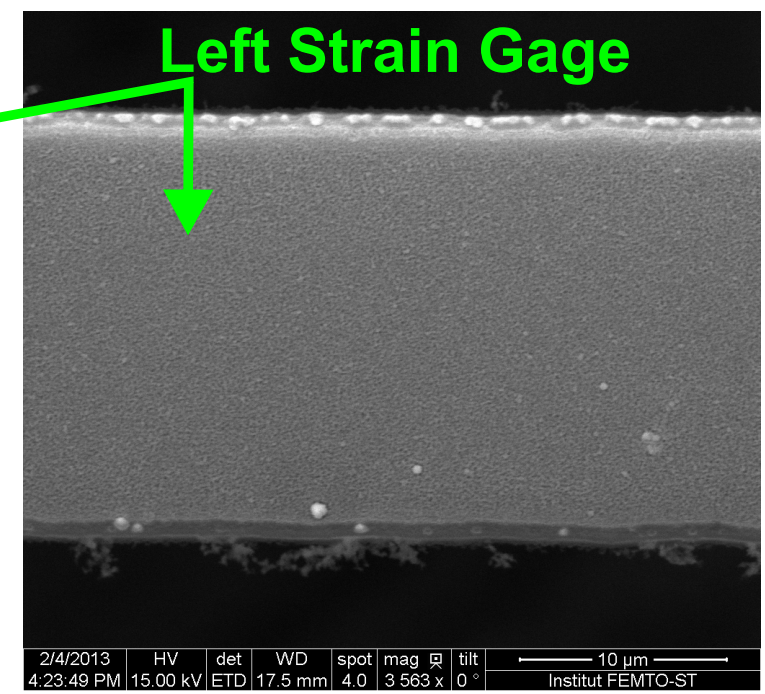

(d)

Figure 6. SEM photos of the three beams structure of the sensing part: (a)\&(b) fixation of the three beam, (c)\&(d) zoom on one beam. (a)\&(c) are side photos while (b)\&(d) are top view.

\subsection{Mechanical Characterization}

The first step of the characterization of the PiezoFS is the mechanical characterization which consists of measuring the stiffness of the PiezoFS. The stiffness measurement is done by moving forward the CalibFS to be in contact with the PiezoFS with a constant velocity and then moving it back. The force measurement is done using the CalibFS and the position measurement is done using the internal sensor of the fine positioning stage. In order to determine the displacement of the PiezoFS, the stiffness of the CalibFS is $1000 \mathrm{~N} / \mathrm{m}$. The displacement of the PiezoFS is calculated by (5):

$$
\delta_{\text {PiezoFS }}=\delta_{S}-\frac{F_{\text {CalibFS }}}{K_{\text {CalibFS }}}
$$




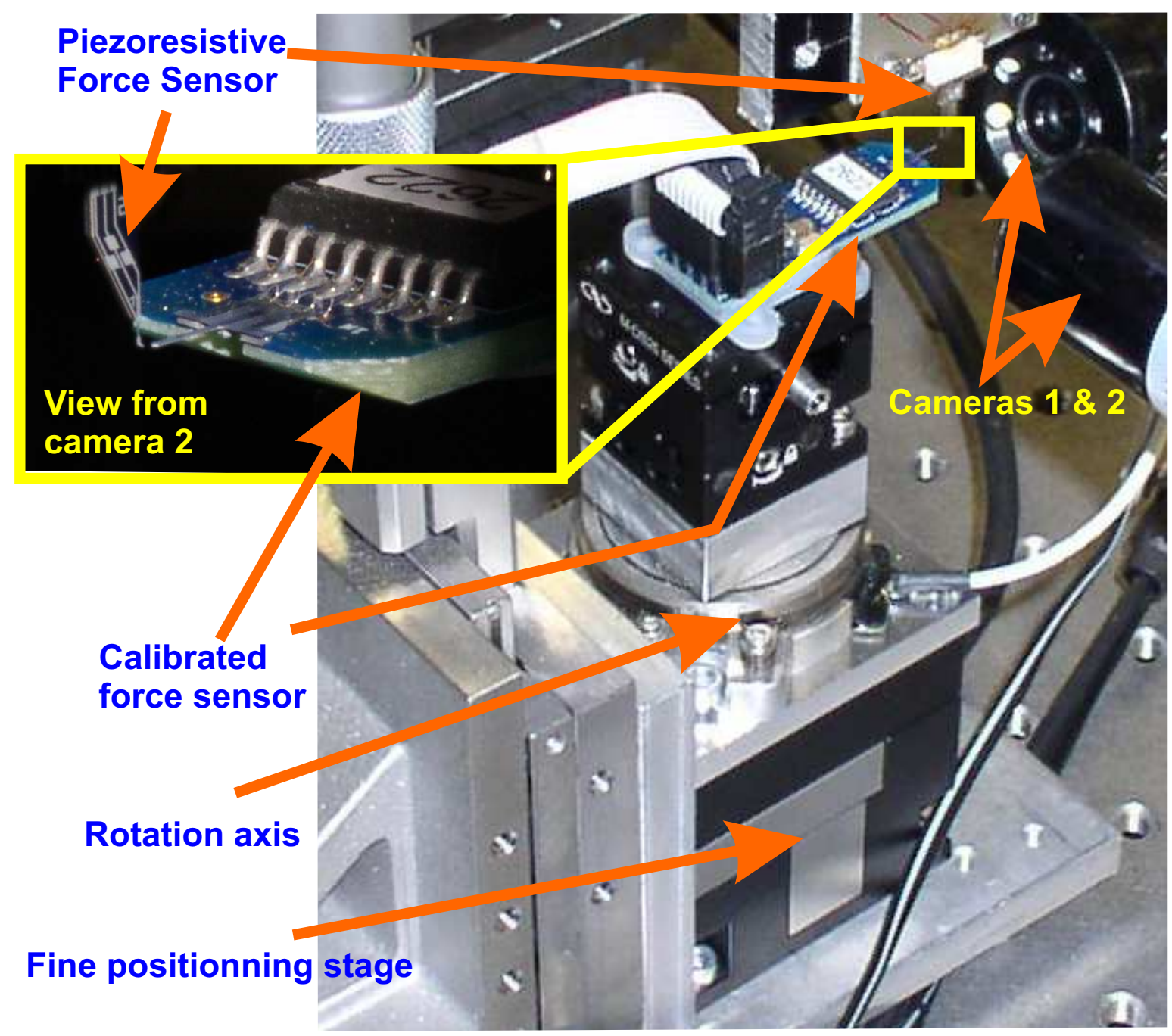

Figure 7. Experimental setup used for the characterization of the piezoresistive force sensor. A calibrated force sensor (CalibFS) is fixed on a robotic system consisting of a fine positioning stage and rotation stage. The piezoresistive force sensor (PiezoFS) is fixed. Two cameras are used to see the contact.

where $\delta_{\text {PiezoFS }}$ is the displacement of the PiezoFS after contact, $\delta_{s}$ is the currently measured position of the internal sensor of the fine positioning stage after contact and $F_{C a l i b F}$ and $K_{\text {CalibFS }}$ are respectively the force measurement signal of CalibFS and its stiffness.

Figure 8 shows the force measurement of the CalibFS with respect to the displacement of the PiezoFS. After contact, the force increases linearly with respect to the displacement. The stiffness is calculated using (6):

$$
K_{\text {PiezoFS }}=\frac{F_{\text {CalibFS }}}{\delta_{\text {PiezoFS }}}
$$

After doing several set of 10 measurements, the stiffness of the force sensor is determined to be $130 \mathrm{~N} / \mathrm{m} \pm 1 \mathrm{~N} / \mathrm{m}$ while the theoretical stiffness is $125 \mathrm{~N} / \mathrm{m}$ which means $4 \%$ of error. 


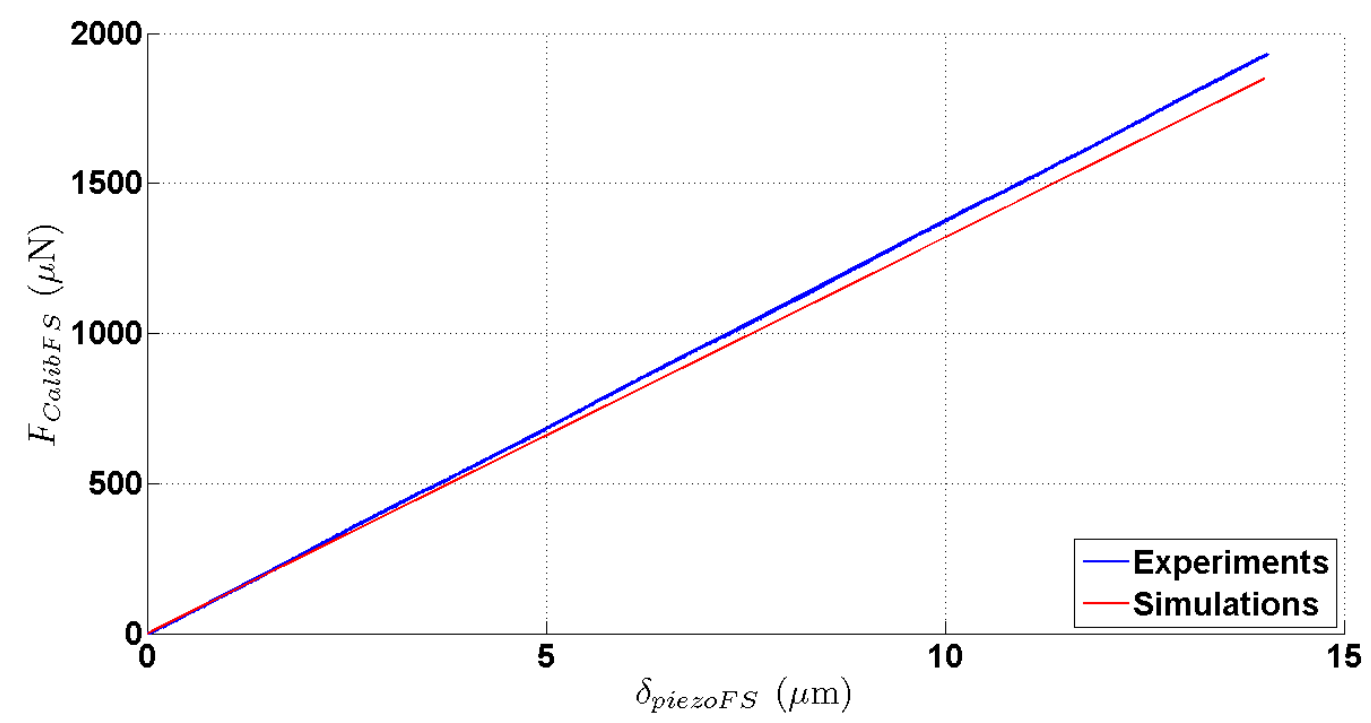

Figure 8. Force measurement of the CalibFS with respect to the position of the fine positioning stage in order to measure the stiffness of the force sensor.

\subsection{Resistance variation and sensor's sensitivity relative to the applied stress}

Without any applied stress on the force sensor, the gage resistance is equal to $R_{1 o}=2.76 \mathrm{k} \Omega$ for one resistance and $R_{2 o}=2.71 \mathrm{k} \Omega$ for the other. The resistance variation of the silicon strain gages is measured while applying a stress on the force sensor. The variation is given in Figure 9-(a). The two strain gages resistances change in push-pull where their variation is linear and symmetric with a relative absolute value of their slope $0.09 \Omega / \mu \mathrm{N}$ for each resistance. The error on the linearity of the resistance variation is less than $0.3 \%$.

Figure 9-(b) shows an example of the readout voltage, $V_{\text {out }}$, at the output of the Wheatstone Bridge circuit supplied with a referenced voltage $V_{r e f}=5 \mathrm{~V}$ and with a differential amplifier composed of a INA103 configured with an amplification factor $A=100$. The voltage variation relative to the force is linear and the measurements are repeatable for several tenth set of measurements done. The average sensitivity of the readout voltage for 10 measurements is $197.5 \mu \mathrm{N} / \mathrm{V}$ for a referenced voltage of $V_{r e f}=5 \mathrm{~V}$. The standard deviation of the 10 measurements with respect to the average is $0.73 \mu \mathrm{N} / \mathrm{V}$. The error on the repeatability of the force sensor is less than $0.3 \%$.

The noise amplitude at the voltage of the PiezoFS is acceptable and has almost the same level of that of the CalibFS. The maximal peak to peak amplitude of noise without contact is around $40 \mathrm{mV}$. The noise amplitude is almost constant for any applied force on the two force sensors. However, the main advantage of the PiezoFS is that it presents much bigger signal to noise ratio (SNR) than the CalibFS. The SNR being dependent of the applied force on the force sensor, the SNR for the PiezoFS reaches $50 \mathrm{~dB}$ at a force of $2 \mathrm{mN}$ where it is $37 \mathrm{~dB}$ for the CalibFS. 

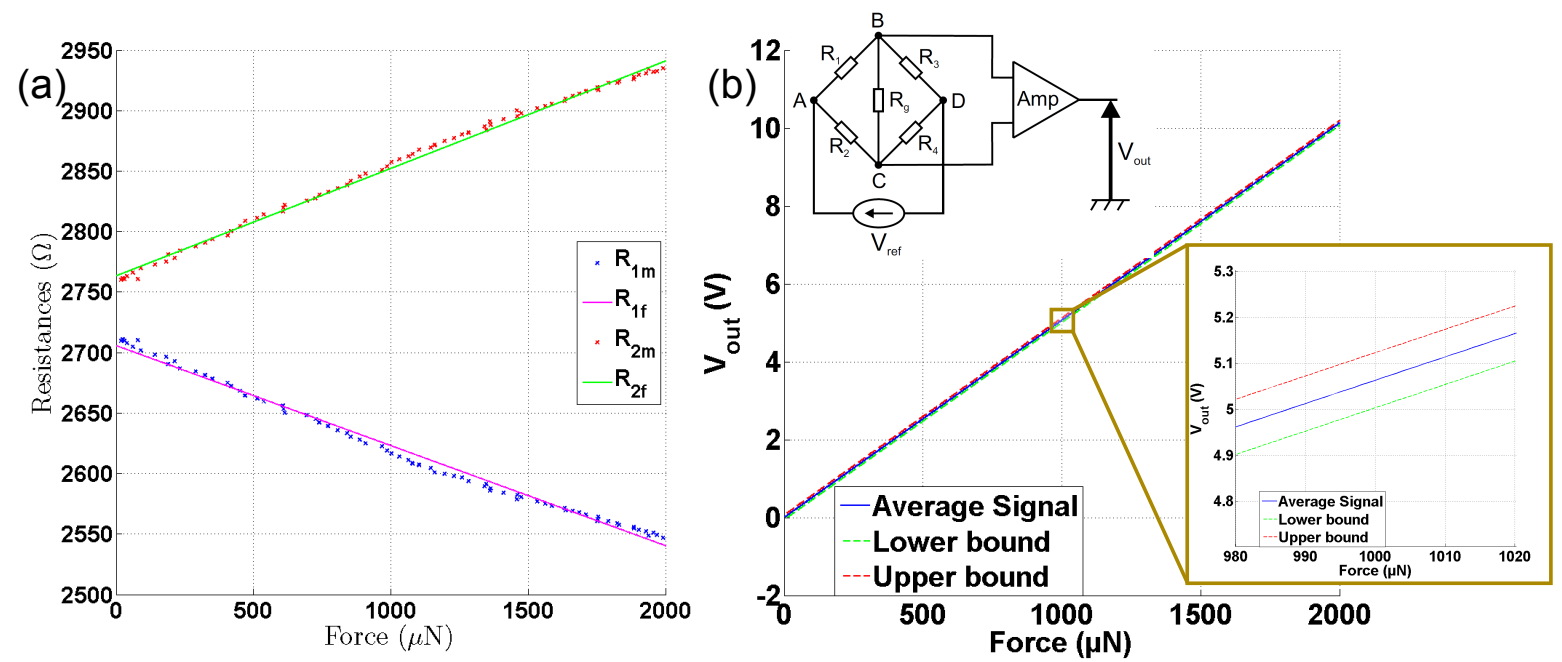

Figure 9. (a) The variation of the strain gages resistances relative to an applied force on the tip of the force sensor. $R_{1 m}$ and $R_{2 m}$ are the measured values of resistances in experiments and $R_{1 f}$ and $R_{2 f}$ are the approximated linear variation of the resistances and (b) The variation of the readout voltage from the Wheatstone Bridge, $V_{\text {out }}$, for a referenced voltage of $V_{\text {ref }}=5 \mathrm{~V}$ and amplification gain, $A=100$, relative to an applied force.

\subsection{Resolution}

The resolution is the smallest reliable force detected by the force sensor. It depends on the bandwidth of the electric circuit and on the filtering. There are many ways of considering the resolution. The first and the worst case is to consider the signal without any filtering on a specific time. In this case, the resolution is equal to the level of noise in the signal (i.e. $8 \mu \mathrm{N}$ ). The second case consists of considering the signal over an interval of time without filtering and then a small variation of force can be detected by the force signal as shown in Figure 10-(a). In Figure 10-(a), the noisy signal of the sensor is shown with respect to the time with the average signal and the upper and lower bounds of the signal which was determined using the filter presented in [34]. A force variation smaller than $1 \mu \mathrm{N}(800 \mathrm{nN})$ is detected. The latter force $(800 \mathrm{nN})$ is smaller than the amplitude of the noise as shown in the figure. The third case consists of filter the signal given by the force sensor over an interval of time. By considering some filtering of the data, a much smaller resolution could be detected by using some signal processing techniques or some advanced filters. The resolution reaches less than $500 \mathrm{nN}$ with a first order low-pass filter with a cut-off frequency of $1 \mathrm{kHz}$ and around $100 \mathrm{nN}$ with a first order low-pass filter with a cut-off frequency of $10 \mathrm{~Hz}$ as shown in Figure 10-(b). The last and ideal case is the theoretical resolution that could obtained by detecting the smallest voltage detection of the data acquisition card. In the case of the study, the data acquisition card is a 16 bits Analog to Digital Converter (ADC) with an input of the card going from -10V to $10 \mathrm{~V}$, the lowest voltage that could be detected is given by the following $\frac{20}{2^{16}} \approx 0.3 \mathrm{mV}$. Then using an appropriate filter the resolution of the force sensor could be improved. The theoretical limit of the resolution is $60 \mathrm{nN}$. 

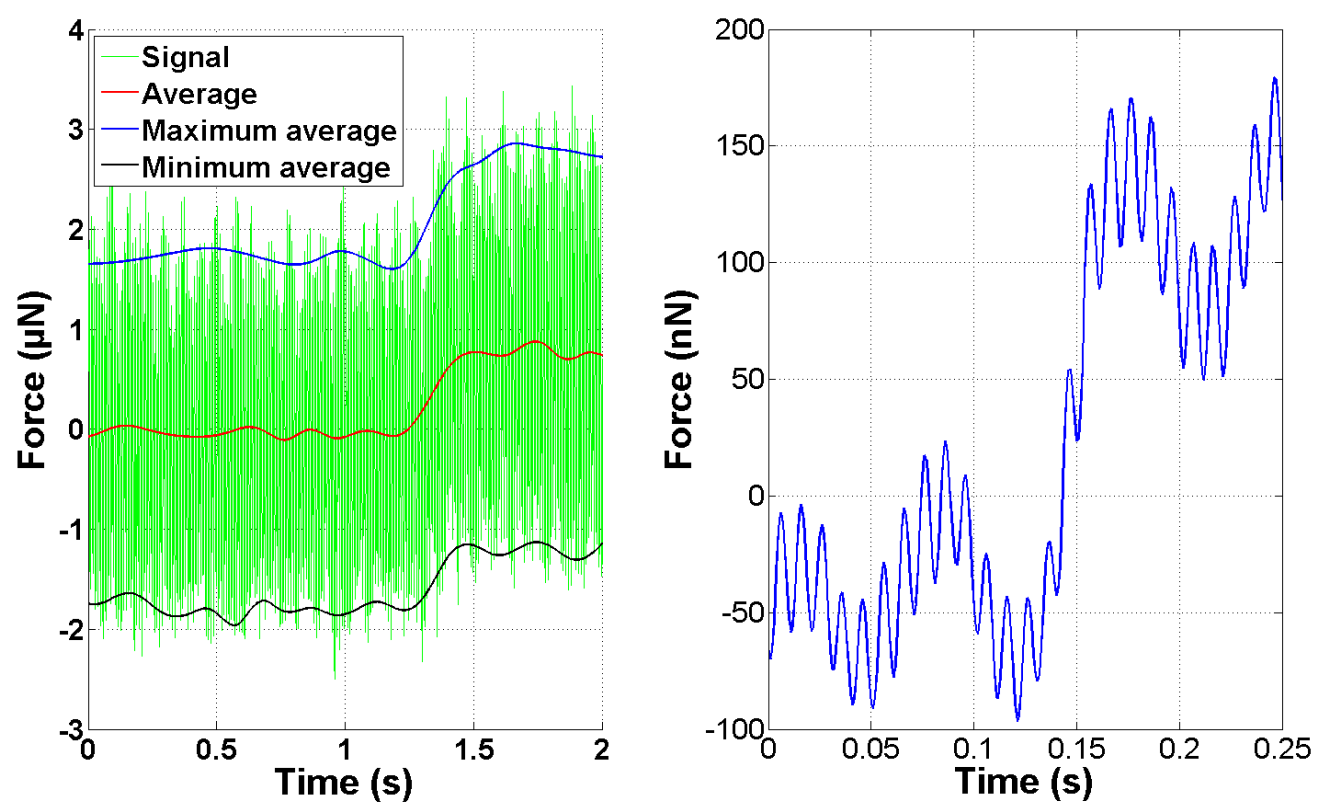

Figure 10. The force sensor signal measured while applying small displacement of the positioning stage in order to determine the resolution of the sensor: (a) resolution of $800 \mathrm{nN}$ without filtering, (b) resolution of $100 \mathrm{nN}$ with a low pass filter with a cut-off frequency of $10 \mathrm{~Hz}$.

\subsection{Discussions about experimental results}

In this section, the experimental results are compared with the design presented in section 2 . The dimensions of the force sensor after the microfabrication have been presented in section 4. The length of the strain gages is $700 \mu \mathrm{m}$, their width is measured to be $20.05 \mu \mathrm{m}$ while in the design it is $20 \mu \mathrm{m}$ and the thickness of the force sensor is measured to be $12.52 \mu \mathrm{m}$ while in the design it is $12 \mu \mathrm{m}$.

The stiffness of the force sensor using the design parameters (i.e. $700 \mu \mathrm{m}$ in length, $20 \mu \mathrm{m}$ in width of the gages and $12 \mu \mathrm{m}$ in thickness) is $125 \mathrm{~N} / \mathrm{m}$. Inducing the experimental dimensions obtained after the microfabrication (i.e. $700 \mu \mathrm{m}$ in length, $20.05 \mu \mathrm{m}$ in width and $12.52 \mu \mathrm{m}$ in thickness instead of $20 \mu \mathrm{m}$ and $12 \mu \mathrm{m}$ respectively) in simulations, the stiffness of the force sensor is calculated to be $130.4 \mathrm{~N} / \mathrm{m}$. In experiments, the stiffness of the PiezoFS is $130 \mathrm{~N} / \mathrm{m}$ which means $0.31 \%$ of error after taking in consideration the dimensions errors in the microfabrication and $4 \%$ of error relative to the initial design.

The gage resistances of the force sensor without any applied stress is set to be $3 \mathrm{k} \Omega$ in design. However, using the experimental results of the dimensions, the gage resistance is calculated to be $2.75 \mathrm{k} \Omega$. The experimental results show that the gage resistances without any stress applied at the tip of the force sensor is $2.8 \mathrm{k} \Omega$ which means $1.8 \%$ of error relative to the resistance taking into consideration the dimensions errors of the microfabrication and $6.7 \%$ of error relative to the design gage resistance. This step shows that the ohmic contact step $\left(3^{r d}\right.$ step of the microfabrication process) is done in a good way and the aluminium has well diffused in 


\begin{tabular}{|c|c|c|c|}
\hline & Model & Results & Error $(\%)$ \\
\hline Length $(\mu \mathrm{m})$ & 700 & 702.8 & 0.4 \\
\hline Beam width $(\mu \mathrm{m})$ & 20 & 20.05 & 0.25 \\
\hline Thickness $(\mu \mathrm{m})$ & 12 & 12.24 & 2 \\
\hline Stiffness $(\mathrm{N} / \mathrm{m})$ & 125 & 130 & 4 \\
\hline Gage resistance $(\mathrm{k} \Omega)$ & 3 & 2.76 & 8 \\
\hline$\Delta \mathrm{R}(\Omega / \mu \mathrm{N})$ & 0.1 & 0.09 & 10 \\
\hline
\end{tabular}

Table 2. Comparison between theoretical model and microfabrication results. The dimensions are measured using SEM and the other variables are measured through experiments. The error is between experimental results and the theoretical parameters with taking into consideration the dimensions error in the microfabrication process.

the silicon.

Once a force is applied to the tip of the force sensor, the left and right resistance change in push-pull. In simulations, the variation of each gage resistance is $0.1 \Omega / \mu \mathrm{N}$ in each direction. While in experiments, the variation of each gage resistance is measured to be $0.09 \Omega / \mu \mathrm{N}$ which means $10 \%$ of error. However, taking into consideration the uncertainities of the microfabrication, the variation of the resistance in simulations was $0.95 \Omega / \mu \mathrm{N}$ which means $5.3 \%$.

Furthermore, theoretical gage factor is bigger than 100 [32] for silicon while in experiments it is estimated to be around 120 which is in the range given by the literature. The gage factor is the $G$ constant given by (2).

These experimental results show that the microfabrication process is reliable. The differences between the model and the results are summarized in Table 2.

\section{Conclusion and future works}

In this paper, the prototyping of a new piezoresistive small microforce sensor has been presented. The design taking advantage of both mechanical and bulk piezoresistive properties of silicon is presented. This force sensor is fabricated using SOI wafer because the use of silicon is widespread in MEMS, it presents a high gage factor and it is fabricated using classical MEMS microfabrication processes. This piezoresistive force sensor has been fabricated using a relatively simple and cheap microfabrication process making it easy to integrate in most of MEMS devices. The sensor also presents high performances compared to the sensors presented in literature thanks to its sensitivity of $197 \mu \mathrm{N} / \mathrm{V}$, its resolution of $100 \mathrm{nN}$, its sensing range of $2 \mathrm{mN}$, its signal to noise ratio of $50 \mathrm{~dB}$ at a force of $2 \mathrm{mN}$ and its stiffness of $130 \mathrm{~N} / \mathrm{m}$. The signal of the force sensor is repeatable with an error less than $0.3 \%$. It was shown that the experimental results are very close to the theoretical results which shows that the process used guarantees the desired behavior of the sensor. The success rate of the force sensor was around $95 \%$ and regarding its small dimensions, many of these sensors could be fabricated on the same SOI wafer which enables to integrate them easily in many MEMS 
structures and offer measurment very close to the area of interest which is a very important issue at microscale.

Hence, based on the obtained results, many force sensors with different sensing ranges and for many different microscale applications can be designed. This paper shows the efficiency of the approach followed for a case study.

This force sensor could be used for micromanipulation by fixing this force sensor on an microgripper finger which enables to control the gripping force applied to the manipulated object.

The characteristics of this force sensor are compatible with many microscale applications such as biological, biomedical, microassembly, micromanipulation, etc. This force sensor range and its resolution help its use for the measurement of some small level forces present at the microscale. Another advantage of this force sensor is that it could be easily integrated into any microsystem due to its small size.

\section{Acknowledgments}

These works have been funded by the Franche-Comté region, partially supported by the Labex ACTION project (contract ANR-11-LABEX-01-01), by the Equipex ROBOTEX project (contract ANR-10-EQPX-44-01) and by the French RENATECH network through its FEMTO-ST technological facility.

\section{References}

[1] R.E. Mackay, H.R. Le, S. Clark, and J.A. Williams. Polymer micro-grippers with an integrated force sensor for biological manipulation. J. Micromech. Microeng., 23, 2013.

[2] M. Himmelhaus and A. Francois. In-vitro sensing of biomechanical forces in live cells by a whispering gallery mode biosensor. Biosensors and Bioelectronics, 25:418427, Oct. 2009.

[3] A. Menciassi, A. Eisinberg, G. Scalari, and C. Anticoli. Force feedback-based microinstrument for measuring tissue properties and pulse in microsurgery. IEEE International Conference on Robotics and Automation (ICRA), 1:626-631, 2001.

[4] J. Agnus, N. Chaillet, C. Clévy, S. Dembélé, M. Gauthier, Y. Haddab, G. Laurent, P. Lutz, N. Piat, K. Rabenorosoa, M. Rakotondrabe, and B. Tamadazte. Robotic microassembly and micromanipulation at femto-st. Journal of Molecular Biology Research (JMBR), 8(2):91-106, 2013.

[5] S. Bargiel, K. Rabenorosoa, C. Clévy, C. Gorecki, and P. Lutz. Towards micro-assembly of hybrid moems components on a reconfigurable silicon free-space micro-optical bench. J. Micromech. Microeng., 20, 2010.

[6] M. Rakotondrabe, C. Clévy, K. Rabenorosoa, and K. Ncir. Presentation, force estimation and control of an instrumented platform dedicated to automated micromanipulation tasks. IEEE International Conference on Automation Science and Engineering (CASE), pages 722 - 727, Toronto, Canada, August 2010.

[7] B. Komati, K. Rabenorosoa, C. Clévy, and P. Lutz. Automated guiding task of a flexible micropart using a two-sensing-finger microgripper. IEEE Transactions on Automation, Science and Engineering, 10, Isue: 3:515 - 524, July 2013.

[8] B. Komati, M.R. Pac, I. Ranatunga, C. Clévy, D. Popa, and P. Lutz. Explicit force control v.s. impedance control for micromanipulation. ASME International Design Engineering Technical Conferences \& Computers and Information in Engineering Conference (IDETC), Portland, USA, August 2013. 
[9] C. Clévy, M. Rakotondrabe, and N. Chaillet. Signal Measurement and Estimation Techniques for Micro and Nanotechnology. Springer, 2011.

[10] M. Boudaoud, Y. Haddab, Y. Le Gorrec, and P. Lutz. Noise characterization in millimeter sized micromanipulation systems. International journal of Mechatronics, 21:1087-1097, 2011.

[11] N. Chaillet and S. Régnier. Microrobotics for Micromanipulation. Wiley-ISTE, 2010.

[12] K. Rabenorosoa, C. Clévy, P. Lutz, M. Gauthier, and P. Rougeot. Measurement of pull-off force for planar contact at the microscale. Micro Nano Letters, 4:148 -154, 2009.

[13] K. Rabenorosoa, C. Clevy, Q. Chen, and P. Lutz. Study of forces during microassembly tasks using twosensing-fingers grippers. IEEE/ASME Transactions on Mechatronics, 17, Issue: 5:811 - 821, Oct. 2012.

[14] Y. Sun, B.J. Nelson, D.P. Potasek, and E. Enikov. A bulk microfabricated multi-axis capacitive cellular force sensor using transverse comb drives. J. Micromech. Microeng., 12, 2002.

[15] E.T. Enikov and B.J. Nelson. Three-dimensional microfabrication for a multi-degree-of-freedom capacitive force sensor using fibre-chip coupling. J. Micromech. Microeng., 10, 2000.

[16] Y. Sun, S.N. Fry, D.P. Potasek, and D.J. Bell. Characterizing fruit fly flight behavior using a microforce sensor with a new comb-drive configuration. Journal of Microelectromechanical Systems, 14 , Issue: 1:4 - 11, Feb. 2005.

[17] F.R. Blom, S. Bouwstra, J.H.J. Fluitman, and M. Elwenspoek. Resonating silicon beam force sensor. Sensors and Actuators, Volume 17, Issues 34:513519, May 1989.

[18] C.J. Van Mullem, F.R. Blom, J.H.J. Fluitman, and M. Elwenspoek. Piezoelectrically driven silicon beam force sensor. Sensors and Actuators A: Physical, Volume 26, Issues 13:379383, March 1991.

[19] Y. Shen, N. Xi, U.C. Wejinya, and W.J. Li. High sensitivity 2-d force sensor for assembly of surface mems devices. Proceedings. IEEE/RSJ International Conference on Intelligent Robots and Systems (IROS 2004), 4:3363 - 3368, 2004.

[20] J. Wang and X. Li. A single-wafer-based single-sided bulk-micromachining technique for high-yield and low-cost volume production of pressure sensors. 16th International Solid-State Sensors, Actuators and Microsystems Conference (TRANSDUCERS'11), pages 410 - 413, June 2011.

[21] H.S. Hsieh, H.C. Chang, C.F. Hu, C.L. Cheng, and W. Fang. A novel stress isolation guard-ring design for the improvement of a three-axis piezoresistive accelerometer. J. Micromech. Microeng., 21, 2011.

[22] D. Li, T. Zhao, Z. Yang, and D. Zhang. Monolithic integration of a micromachined piezoresistive flow sensor. J. Micromech. Microeng., 20, 2010.

[23] M. Tortonese, R.C. Barrett, , and C.F. Quate. Atomic resolution with an atomic force microscope using piezoresistive detection. Appl. Phys. Lett., 62, no. 8:834836, 1993.

[24] A. Wisitsoraat, V. Patthanasetakul, T. Lomas, and A. Tuantranont. Low cost thin film based piezoresistive mems tactile sensor. Sensors and Actuators A, 139:1722, 2007.

[25] M. Li, H.X. Tang, and M.L. Roukes. Ultrasensitive nems-based cantilevers for sensing, scanned probe and very-high frequency applications. Nature Nanotechnology, 2:114120, 2007.

[26] J. Wei, S. Magnani, and P.M. Sarro. Suspended submicron silicon-beam for high sensitivity piezoresistive force sensing cantilevers. Sensors and Actuators A: Physical, 186:8085, 2012.

[27] J.C. Doll and B.L. Pruitt. High-bandwidth piezoresistive force probes with integrated thermal actuation. J. Micromech. Microeng., 22, 2012.

[28] K. Mølhave and O. Hansen. Electro-thermally actuated microgrippers with integrated force-feedback. $J$. Micromech. Microeng., 15, 2005.

[29] L. Liu, S. Pal, and H. Xie. Mems mirrors based on a curved concentric electrothermal actuator. Sensors and Actuators A: Physical, 188:349358, 2012.

[30] C. Liu. Foundations of MEMS. Pearson Education, 2012.

[31] S.U. Jen, C.C. Yu, C.H. Liu, and G.Y. Lee. Piezoresistance and electrical resistivity of pd, au, and cu films. Thin Solid Films, 434:316-322, 2003.

[32] J. Thaysen, A. Boisen, O. Hansen, and S. Bouwstra. Atomic force microscopy probe with piezoresistive read-out and a highly symmetrical wheatstone bridge arrangement. Sensors and Actuators A: Physical, 83:47-53, 2000.

[33] D. Hériban and M. Gauthier. Robotic micro-assembly of microparts using a piezogripper. In Proc. of the 
2008 IEEE/RSJ International Conference on Intelligent Robots and Systems, pages 4042 - 4047, Nice, France, 2008.

[34] A. Komaty, A.O. Boudraa, B. Augier, and D. Dare-Emzivat. Emd-based filtering using similarity measure between probability density functions of imfs. IEEE Transactions on Instrumentation and Measurement, 63, no.1:27-34, Jan. 2014. 